\title{
Hyménoptères parasites (Ichneumonoidea) capturés au piège à vitre dans le domaine du Rond-Chêne (Esneux, Province de Liège) et focus sur 3 espèces de Xorides Latreille (Ichneumonidae : Xoridinae) présents en Belgique
}

\author{
Pierre-Nicolas Libert ${ }^{(1)} \&$ Fons Verheyde ${ }^{(2)}$ \\ ${ }^{(1)}$ Collaborateur scientifique pour Université de Liège, Gembloux Agro-Bio Tech, Unité d'Entomologie \\ fonctionnelle et évolutive (Prof. Frédéric Francis). Passage des Déportés 2, B-5030 Gembloux (Belgium). \\ Correspondance personnelle : rue de Ramezée, 8, B-5377 Somal (Belgium). \\ E-mail : libert-danckers@skynet.be
}

\author{
${ }^{(2)}$ Aartshertoginnestraat 58/01, B-8400 Ostend, Belgium \\ E-mail : fonsverheyde@hotmail.com
}

Reçu le 14 avril 2021, accepté le 03 juin 2021

\begin{abstract}
Neuf espèces d'Hyménoptères parasites (Braconidae, Ichneumonidae) sont signalées pour la première fois en Belgique: Atanycolus denigrator (Linnaeus 1758), Cosmophorus regius Niezabitowski 1910, Aleiodes dissector (Nees, 1834), Rhimphoctona pectoralis (Kriechbaumer, 1890), Rhimphoctona rufocoxalis (Clément, 1924), Rhimphoctona xoridiformis (Holmgren, 1860), Xorides alpestris (Habermehl, 1903), Xorides brachylabis (Kriechbaumer, 1889) et Xorides rufipes (Gravenhorst, 1829). Une note détaillée est fournie pour chaque espèce. Une carte de répartition est présentée pour les espèces du genre Xorides Latreille.

Mots-clés : Braconidae, Ichneumonidae, Atanycolus, Cosmophorus, Aleiodes, Rhimphoctona, Xorides, données faunistiques, nouvelles occurrences, Rond-Chêne, Belgique.
\end{abstract}

Nine species of parasitic Hymenoptera (Braconidae, Ichneumonidae) are recorded for the first time in Belgium: Atanycolus denigrator (Linnaeus 1758), Cosmophorus regius Niezabitowski 1910, Aleiodes dissector (Nees, 1834), Rhimphoctona pectoralis (Kriechbaumer, 1890), Rhimphoctona rufocoxalis (Clément, 1924), Rhimphoctona xoridiformis (Holmgren, 1860), Xorides alpestris (Habermehl, 1903), Xorides brachylabis (Kriechbaumer, 1889) et Xorides rufipes (Gravenhorst, 1829). Detailed informations are given for each species. A distribution map is presented for Xorides Latreille species.

Key-words : Braconidae, Ichneumonidae, Atanycolus, Cosmophorus, Aleiodes, Rhimphoctona, Xorides, faunistic data, new records, Rond-Chêne, Belgium. 


\section{INTRODUCTION}

Nous avons eu l'occasion d'examiner des reliquats de captures aux pièges à vitre réalisées dans le Domaine du Rond-Chêne situé sur les hauteurs d'Esneux en Province de Liège. Ces pièges, initialement installés afin de récolter des Scolytes (Coleoptera : Curculionidae : Scolytinae), ont également permis la capture d'autres insectes. Parmi ceux-ci des Hyménoptères parasitoïdes appartenant aux familles des Braconidae et des Ichneumonidae (Hymenoptera : Ichneumonoidea). Le Domaine du Rond-Chêne est un vaste parc de 35 ha comprenant un château, un arboretum et des parcelles boisées ; il appartient à la Fédération WallonieBruxelles et sert de centre de dépaysement scolaire.

\section{MATÉRIEL et MÉTHODE}

La campagne de piégeage s'est déroulée entre le 15 avril et le 30 juin 2020 dans l'enceinte du Domaine du Rond-Chêne. Des pièges à vitre ont été installés, certains à proximité d'un vieil épicéa abattu (Picea abies (L.) H. Karst 1881) (Figure 1). Les Hyménoptères capturés ont été triés et les parasitoïdes nous ont été soumis en alcool.

Parmi les espèces rencontrées, plusieurs appartenaient au genre Xorides Latreille 1809 (Ichneumonidae : Xoridinae). Il nous a semblé utile, pour ces espèces, de faire le bilan des captures effectuées en Belgique ces dernières années. En effet, le premier auteur a revu pour ce groupe, l'ensemble des collections entomologiques d'Ichneumonidae dans les institutions belges: ULiège-Gembloux-Entomologie fonctionnelle et évolutive (Gembloux), ULiège-Musée de Zoologie (Liège), ULiège-Station scientifique des Hautes-Fagnes (Mont Rigi), Musée de Zoologie (Mons), Centre wallon de Recherches agronomiques (Gembloux), Institut royal des Sciences naturelles de Belgique (Bruxelles), Institut provincial d'Enseignement agronomique (La Reid), Haute-École provinciale du Hainaut - Condorcet (Ath), UMonsLaboratoire de Zoologie (Mons), ainsi que les collections privées de Luc Crevecoeur, Philippe Wegnez, Jorgen Ravoet, Jean-Luc Vago, Arnaud Henrard, Jean-Yves Baugnée et Geoffrey Miessen. Le second auteur, quant à lui, analyse, depuis plusieurs années, les données de la plateforme d'observations naturalistes "Observations.be », issue d'une collaboration entre Natuurpunt et Natagora. Par ailleurs, certains des spécimens utilisés ici, proviennent de ses propres captures. 


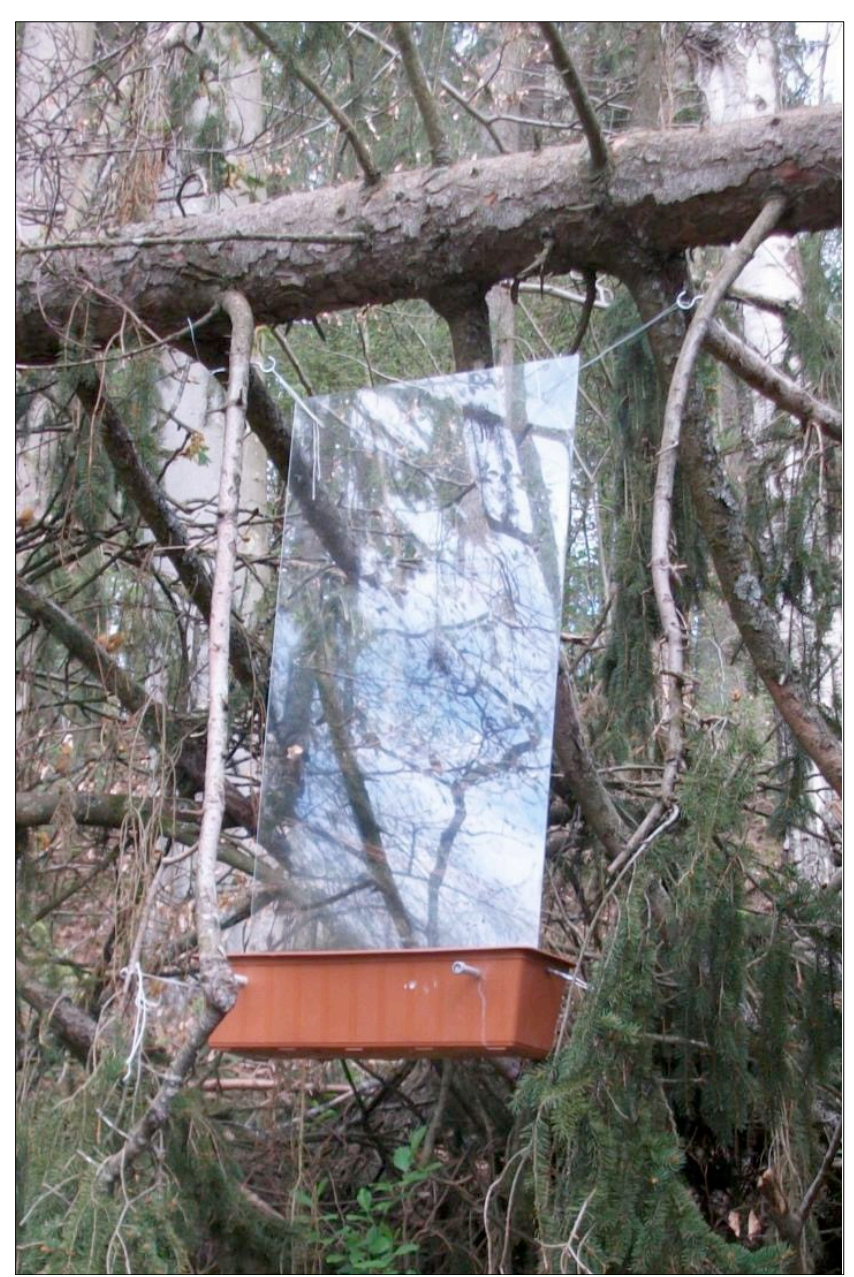

Figure 1 : Piège à vitre installé au pied d'un épicéa abattu du 15 au 30 juin 2020 dans le parc du Domaine du Rond-Chêne à Esneux. (Cliché : (C) Bernard Moucheron)

\section{RÉSULTATS}

Dans les matériaux soumis en provenance du Domaine du Rond-Chêne, très peu d'Ichneumonoidea (Hymenoptera) étaient présents, ce type de piège n'étant pas adapté à leur capture. Parmi ceux-ci, nous avons pu identifier 12 espèces : 4 Braconidae et 8 Ichneumonidae. Neuf sont nouvelles pour la Belgique. Le détail des identifications est repris ci-dessous avec une note pour chaque espèce. Une carte de répartition en Belgique est fournie pour les espèces du genre Xorides Latreille.

\section{Famille des Braconidae Sous-famille des Braconinae}

\section{Atanycolus denigrator (Linnaeus 1758) Belg. sp. nov. (Figure 2)}

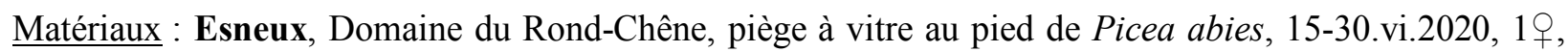
Libert det.

Note : A ce jour, une seule espèce du genre était connue en Belgique : Atanycolus initiator (Fabricius 1793) (Libert \& van Achterberg, 2004). Cette deuxième espèce est connue pour parasiter des Coléoptères Cerambycidae et Buprestidae (Yu et al., 2012) lesquels vivent aux dépends de l'épicéa (van Achterberg, in litt.). 


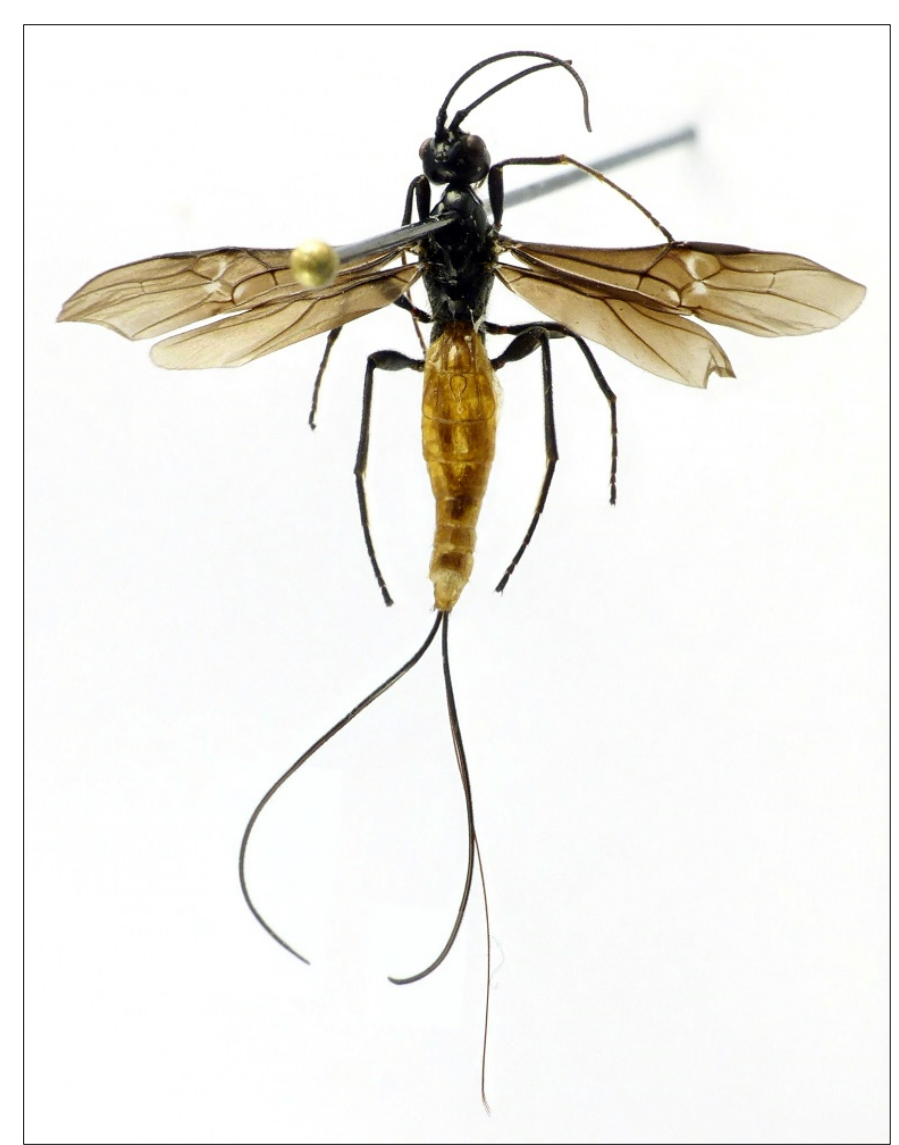

Figure 2 : Atanycolus denigrator (Linnaeus 1758), habitus femelle. (Cliché : (C) Jean-Luc Vago)

\section{Sous-famille des Euphorinae}

2. Cosmophorus regius Niezabitowski 1910 Belg. sp. nov. (Figure 3, a et b)

Matériaux : Esneux, Domaine du Rond-Chêne, piège à vitre au pied de Picea abies, 15-30.vi.2020, 19, Libert det.

Note : Le genre Cosmophorus Ratzeburg compte cinq espèces en Europe (van Achterberg, 2013). Il n'avait jamais été signalé en Belgique. Ce sont des parasites de Scolytes (Coleoptera : Curculionidae : Scolytinae) (Yu et al., 2012). Leur morphologie particulière est adaptée à leur manière de parasiter. En effet, les mandibules outrancièrement développées (Figure 2b) associées à la base des antennes laquelle présente des projections arrondies, permet à l'insecte de saisir le scolyte au niveau du scutellum et de le maintenir ainsi « emprisonné » pendant la ponte (Hedqvist, 1998). En Europe, les hôtes connus sont les Scolytinae Dryocoetes hectographus Reitter 1913, Ips amitinus Eichhoff 1871, Polygraphus poligraphus (Linnaeus 1758), Trypodendron lineatum (Olivier 1795) (Čapek, 1958 ; Hedqvist 1998). Dans le cadre de la présente campagne de piégeage, onze espèces de scolytes ont été capturées. Parmi les espèces présentes sur le site, seule Polygraphus poligraphus (Linnaeus 1758) fait partie des hôtes connus ; seuls cinq individus ont été capturés. Par contre, l'espèce présente en plus grand nombre était Crypturgus subcribrosus Eggers 1933 avec un total de 360 individus sur 408 (Moucheron, in litt.) mais elle ne fait par contre pas partie de la liste des hôtes référencés. Espèce à répartition paléarctique, en Europe elle est connue des pays suivants : Allemagne, Autriche, Finlande, Hongrie, Norvège, Pologne, République Tchèque, Russie, Slovaquie, Suède, Suisse, ex-Yougoslavie (Yu et al., 2012 ; Staverløkk et al., 2020) 


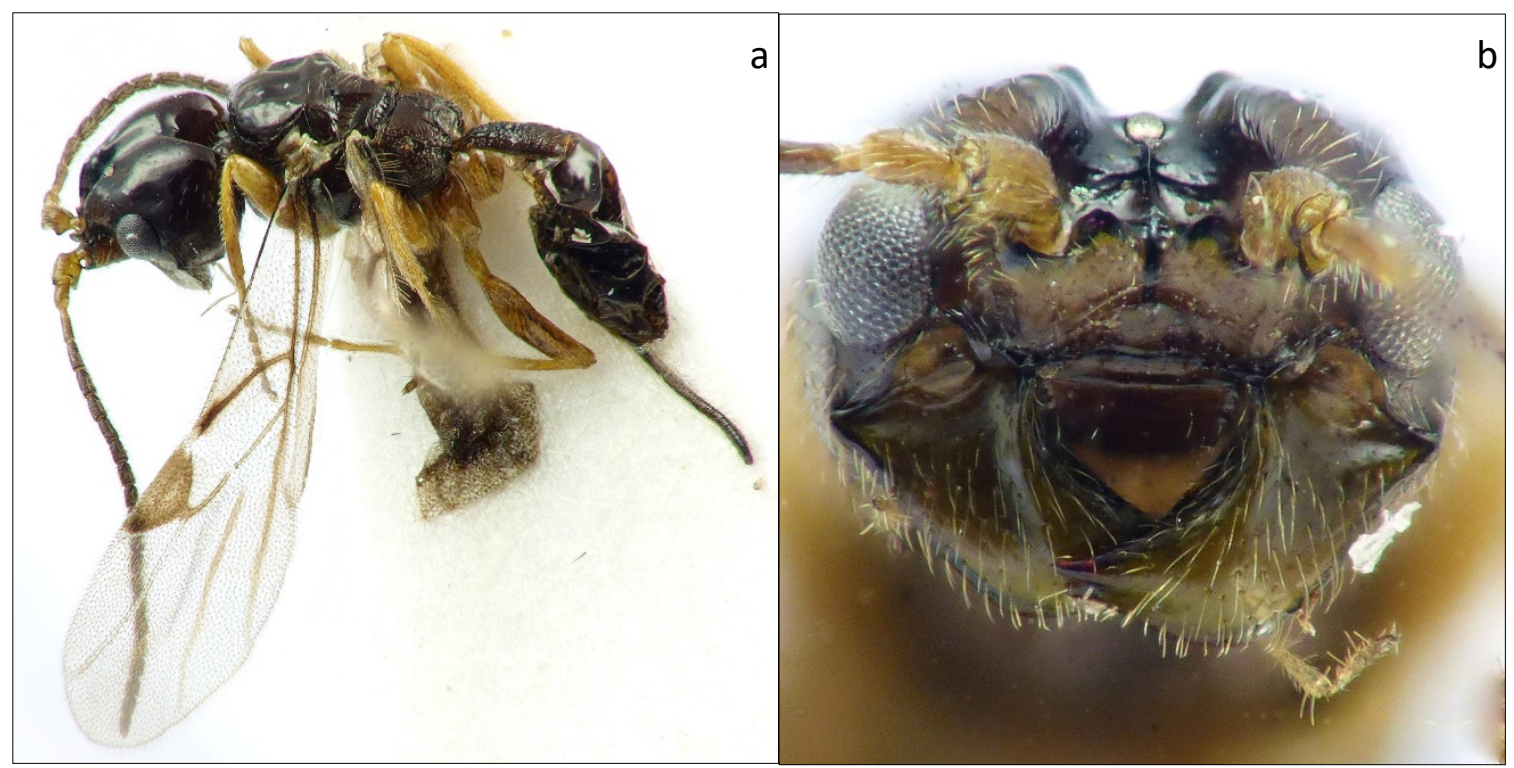

Figure 3 : Cosmophorus regius Niezabitowski 1910, a : habitus femelle ; b : tête vue de face. (Cliché : ( $)$ Jean-Luc Vago)

\section{Sous-famille des Helconinae}

\section{Helconidea dentator (Fabricius 1804)}

Matériaux : Esneux, Domaine du Rond-Chêne, piège à vitre au pied de Picea abies, 15-30.vi.2020, 19, Libert det.

Note : Déjà connu de Belgique, c'est un parasite bien connu de Coléoptères Cerambycidae, notamment du genre Tetropium sp. (van Achterberg, 1987 ; Yu et al., 2012), tout comme Rhimphoctona xoridiformis (Holmgren, 1860) et Xorides brachylabis (Kriechbaumer, 1889) (voir plus loin). Parmi les Cerambycidae du genre capturés au même endroit, seul Tetropium fuscum (Fabricius) était présent (B. Moucheron, in litt.).

\section{Sous-famille des Rogadinae}

\section{4. $\quad$ Aleiodes dissector (Nees, 1834) Belg. sp. nov.}

Matériaux : Esneux, Domaine du Rond-Chêne, piège à vitre au pied de Picea abies, 15-30.vi.2020, 1 , Libert det.

Note : Bien que cité par Marshall (1888) et von Dalla Torre (1898) au $19^{\text {ème }}$ siècle comme faisant partie de la faune belge, la présence de l'espèce sur notre territoire était douteuse. En effet, la récente révision du genre Aleiodes Wesmael a montré que les identifications anciennes devaient faire l'objet de la plus grande prudence car, et nous les citons : «Indeed, we have noted a high level of previous misidentifications in the material we have been able to examine, ...» (van Achterberg \& Shaw, 2016). Il s'agit ici d'une capture opportuniste car elle n'est en rien liée à la proximité de l'épicéa. A. dissector est univoltine et l'espèce est le plus souvent capturée entre mai et juin en milieu forestier feuillu; la larve se développe aux dépends de Lépidoptères Noctuidae du genre Orthosia sp. (van Achterberg et al., 2020). Comme le montre les observations sur les plateformes d'observations naturalistes, cette espèce se rencontre plus en plus fréquemment ces dernières années.

\section{Famille des Ichneumonidae} Sous-famille des Camplopleginae

Le genre Rhimphoctona Förster compte 10 espèces en Europe (van Achterberg, 2013 ; Varga, 2017) dont 5 sont présentes en Belgique: Rhimphoctona grandis (Boyer de Fonscolombe, 1852), Rhimphoctona 
megacephalus (Gravenhorst, 1829), Rhimphoctona melanura (Holmgren, 1860), Rhimphoctona obscuripes (Holmgren, 1860) et Rhimphoctona teredo (Hartig, 1847) (Thirion, 2005). Une révision du genre a été publiée par Horstmann (1980) et une clé récente des espèces européennes par Varga (2017).

\section{Rhimphoctona pectoralis (Kriechbaumer, 1890) Belg. sp. nov. (Figure 4)}

Matériaux : Esneux, Domaine du Rond-Chêne, piège à vitre au pied de Picea abies, 15-30.vi.2020, 1ठ, Libert det.

Note: Les mâles sont facilement reconnaissables à leurs coxas postérieures rouges (noires chez les femelles), la face jaune ainsi que les mésopleures ventralement. Il est cité comme parasite du Cerambycidae Molorchus minor (Linnaeus, 1758), espèce polyphage sur conifères, notamment l'épicéa (Horstmann, 1980 ; Villiers, 1978). On la rencontre en Allemagne, Autriche, Finlande, France, Italie, Pologne et Ukraine (Yu et al. 2012, Varga, 2017).

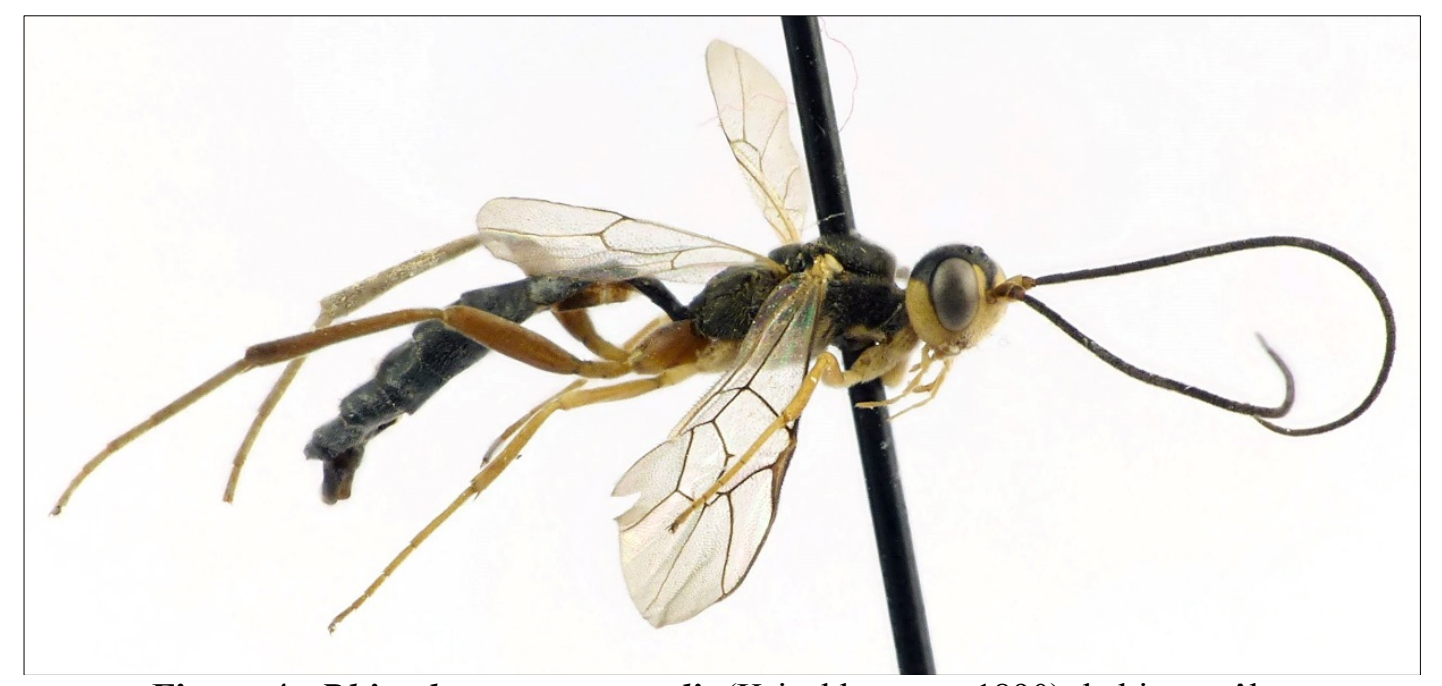

Figure 4 : Rhimphoctona pectoralis (Kriechbaumer, 1890), habitus mâle.

(Cliché : (C) Jean-Luc Vago)

\section{Rhimphoctona rufocoxalis (Clément, 1924) Belg. sp. nov.}

Matériaux : Esneux, Domaine du Rond-Chêne, piège à vitre au pied de Picea abies, 15-30.vi.2020, 19, Libert det.

Note : Les femelles se reconnaissent notamment à leurs coxas postérieures rouges et à la longueur de leur ovipositeur qui égale celle des tibias postérieurs. En Chine, où l'espèce est également présente, elle parasite le Cerambycidae Tetropium castaneum (Linnaeus, 1758) (Luo \& Sheng, 2010). Ce longicorne est également bien présent chez nous (Muylaert, 1990) ; c'est une espèce polyphage sur conifères, notamment l'épicéa (Villiers, 1978). En Europe, R. rufocoxalis est connu des pays suivants : Allemagne, Autriche, France, Italie, Pologne, Roumanie, Russie, Suède, Suisse, ex-Tchécoslovaquie et Ukraine (Yu et al. 2012, Varga, 2017).

\section{Rhimphoctona xoridiformis (Holmgren, 1860) Belg. sp. nov.}

Matériaux : Esneux, Domaine du Rond-Chêne, piège à vitre au pied de Picea abies, 1-15.vi.2020, 10 , Libert det.

Note : Les mâles sont identifiables par leurs coxas postérieures noires, la face jaune avec une ligne noire médiane, l'absence de costula sur le propodeum (parfois légèrement distincte), les fémurs postérieurs rouges ainsi que par les tempes élargies. Ses hôtes les plus probables sont également des Cerambycidae appartenant aux espèces suivantes : Arhopalus rusticus (Linnaeus, 1758), Pogonocherus fasciculatus (De Geer, 1775), Pyrrhidium sanguineum (Linnaeus, 1758), Tetropium fuscum (Fabricius, 1787) et Tetropium gabrieli Weise, 1905 (Horstmann, 1980 ; Uhthoff-Kaufmann, 1990a, 1990b, 1991). Plusieurs d'entre-elles 
sont liées aux conifères (Villiers, 1978) et sont présentes en Belgique (Muylaert, 1990). Néanmoins, parmi les Cerambycidae capturés au même endroit, seul Tetropium fuscum (Fabricius) était présent (B. Moucheron, in litt.). En Europe, l'espèce est connue des pays suivants : Allemagne, Autriche, Finlande, France, Grande-Bretagne, Hongrie, Italie, Norvège, Pologne, Roumanie, Russie, Suède, Suisse, exTchécoslovaquie et Ukraine (Yu et al. 2012, Varga, 2017).

\section{Sous-famille des Pimplinae}

\section{Dolichomitus mesocentrus (Gravenhorst, 1829)}

Matériaux : Esneux, Domaine du Rond-Chêne, piège à vitre au pied de Picea abies, 1-15.v.2020, 1 9 , Libert det.

Note : Il s'agit de l'espèce du genre la plus fréquente en Belgique (Libert, données non publiées). Connue de Belgique depuis les travaux de Jacobs et Tosquinet (1897).

\section{Sous-famille des Xoridinae}

\section{Ischnoceros rusticus (Geoffroy, 1785)}

Matériaux : Esneux, Domaine du Rond-Chêne, piège à vitre au pied de Picea abies, 15-30.vi.2020, 10̂, Libert det.

Note : Deux espèces du genre Ischnoceros se rencontrent en Europe : Ischnoceros caligatus (Gravenhorst, 1829) et Ischnoceros rusticus (Geoffroy, 1785) (van Achterberg, 2013). Toutes deux sont également présentes en Belgique, la première étant moins fréquente que la seconde (Libert, données non publiées). Parmi les Xoridinae, le genre et les deux espèces sont aisément identifiables avec la clé récente de Varga (2014a). Ischnoceros rusticus (Geoffroy, 1785) est connu pour parasiter différentes espèces de Coléoptères Cerambycidae dont plusieurs se rencontrent en Belgique : Aromia moschata Linnaeus, Leiopus nebulosus Linnaeus, Phymatodes testaceus Linnaeus, Rhagium bifasciatum Fabricius, *Rhagium inquisitor Linnaeus, *Rhagium mordax De Geer, Saperda scalaris Linnaeus (Šedivý J., 1958 ; Muylaert, 1990 ; UhthoffKaufmann, 1990b; Campadelli \& Scaramozzino, 1994). Parmi les Cerambycidae capturés au même endroit, seuls ceux précédés d'un «*» étaient présents (B. Moucheron, in litt.)

\section{Xorides alpestris (Habermehl, 1903) Belg. sp. nov. (Figure 5 et 6)}

Matériaux : Esneux, Domaine du Rond-Chêne, piège à vitre au pied de Picea abies, 15-31.v.2020, 1웅 Libert det.

Autres données: Arendonk, 9.ix.2020, 1q, Verheyde det.; ibidem, sur Pinus sp., 12.ix.2020, 1q, Verheyde det. ; Auderghem, Jardin Massart, piège Malaise, 17-24.vi.2017, 10 , Libert det. ; ibidem, 24.v1.vi.2017, 1 , Libert det; Beernem, sur Fagus sylvatica, 17.v.2020, 10, Verheyde \& Varkonyi det.; ibidem, sur Betula sp., 26.v.2020, 1ð, De Ketelaere det. ; ibidem, sur Fagus sylvatica, 1.vi.2020, 1ठ, De Ketelaere \& Verheyde det. ; Bonheiden, 15.ix.2012, 1, Verheyde det. ; ibidem, 26.ix.2013, 1ㅇ, Verheyde det. ; ibidem, 1.x.2013, 1q, Verheyde det. ; Bruxelles, 24.vii.2018, 1q, Verheyde det. ; Corroy-leChâteau, sur Salix sp., 22.vi.1953, 1q, Libert det.; Fraipont, 16.v.2003, 1ठ, Libert det.; Gelrode, 9.vii.2018, 1ㅇ, Verheyde det.; Genk, 15.vii.2020, 19, Verheyde det. ; Hoboken, sur Fagus sylvatica, 8.viii.2018, 1q, Verheyde det.; Gesves, 23.viii.1994, 1q, Libert det.; Houthalen, 1.vi.2020, 1 , Verheyde det. ; Lavaux-Saint-Anne, 19.v.2007, 1q, Libert det. ; Lozer, 27.viii.2019, 1 q, Verheyde det. ; ibidem, 15.ix.2020, 19, Verheyde det.; Malderen, 15.viii.2015, 1q, Verheyde det. ; Malonne, 18.vii.2007, 19, Libert det.; Meeuwen, Dorpenmolen, piège Malaise, 1-9.vi.2009, $1 \widehat{O}^{\mathrm{O}}$, Libert det. ; Merelbeke, 27.v.2012, 1q, Verheyde det.; Mol, sur Betula sp., 4.viii.2020, 1q, Verheyde det. ; Nazareth, 24.vii.2018, 19, Verheyde det.; Purnode, 22.vii.1980, 1q, Libert det. ; Retie, 27.v.2020, 19, Verheyde det. ; Scherpenheuvel-Zichem, 7.vii.2019, 1क, Verheyde det. ; Somal, sur Acer sp., bordure de l'Eau de Somme, 27.v.2020, 1ð̂, Libert det.; Spa, 8.vii.2020, 1q, Verheyde det.; Stambruges, Mer de sable, 14.vii.2014, 1q, Verheyde det.; Tessenderlo, 22.vii.2018, 1q, Verheyde det.; Testelt, 26.vi.2018, 1 , 
Verheyde det. ; Trois-Ponts, Fosse, 31.v.2018, 1q, Verheyde det. ; Woumen, sur Salix sp., 3.ix.2020, 1, Verheyde det. ; Zillebeke, sur Fagus sylvatica, 23.v.2020, 10,, Verheyde \& Varkonyi det.

Note : La première attestation de présence de Xorides alpestris (Habermehl, 1903) en Belgique date de 1953 ; il s'agissait d'un spécimen femelle capturé à Corroy-le-Château sur un saule (collection ULiègeGembloux). Néanmoins les données concernant cette espèce restent très éparses jusqu'aux années 2010 où elle semble être cantonnée dans le sud du pays. A partir de 2010 et jusqu'aujourd'hui, son expansion est notable sur tout le territoire et en particulier dans le nord du pays (Figure 5). Ceci est probablement lié à l'augmentation de la présence de bois mort, notamment dans les réserves naturelles (Verheyde et al., 2020). En effet, comme tous les Xoridinae, l'espèce est parasite d'insectes saproxylophages. En l'occurrence, l'espèce est connue pour parasiter des Coléoptères Cerambycidae dont un, au moins est présent, en Belgique : Leptura aethiops Poda (Muylaert, 1990; Hilszczanski, 2002). L'espèce est de répartition paléarctique et, en Europe, elle est présente dans les pays suivants: Allemagne, Autriche, Biélorussie, Bulgarie, Croatie, Finlande, Hongrie, Pologne, République tchèque, Roumanie, Russie, Suède, Suisse, Ukraine et ex-Yougoslavie (Yu et al., 2012; Varga, 2014b). Tout récemment, elle a été découverte en France (Penigot \& Mallet, 2020).

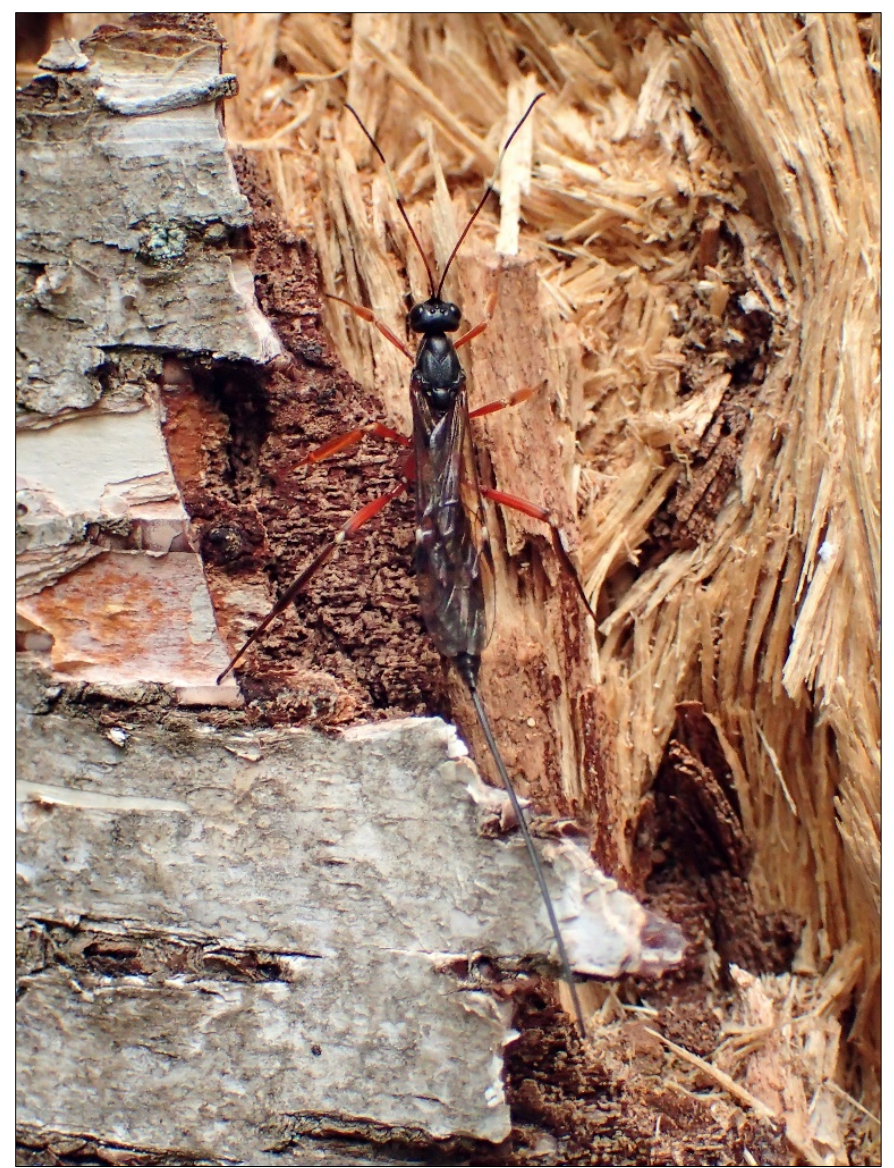

Figure 5 : Xorides alpestris (Habermehl, 1903), habitus femelle. (Cliché : (C) Stefan Verheyen, observations.be) 


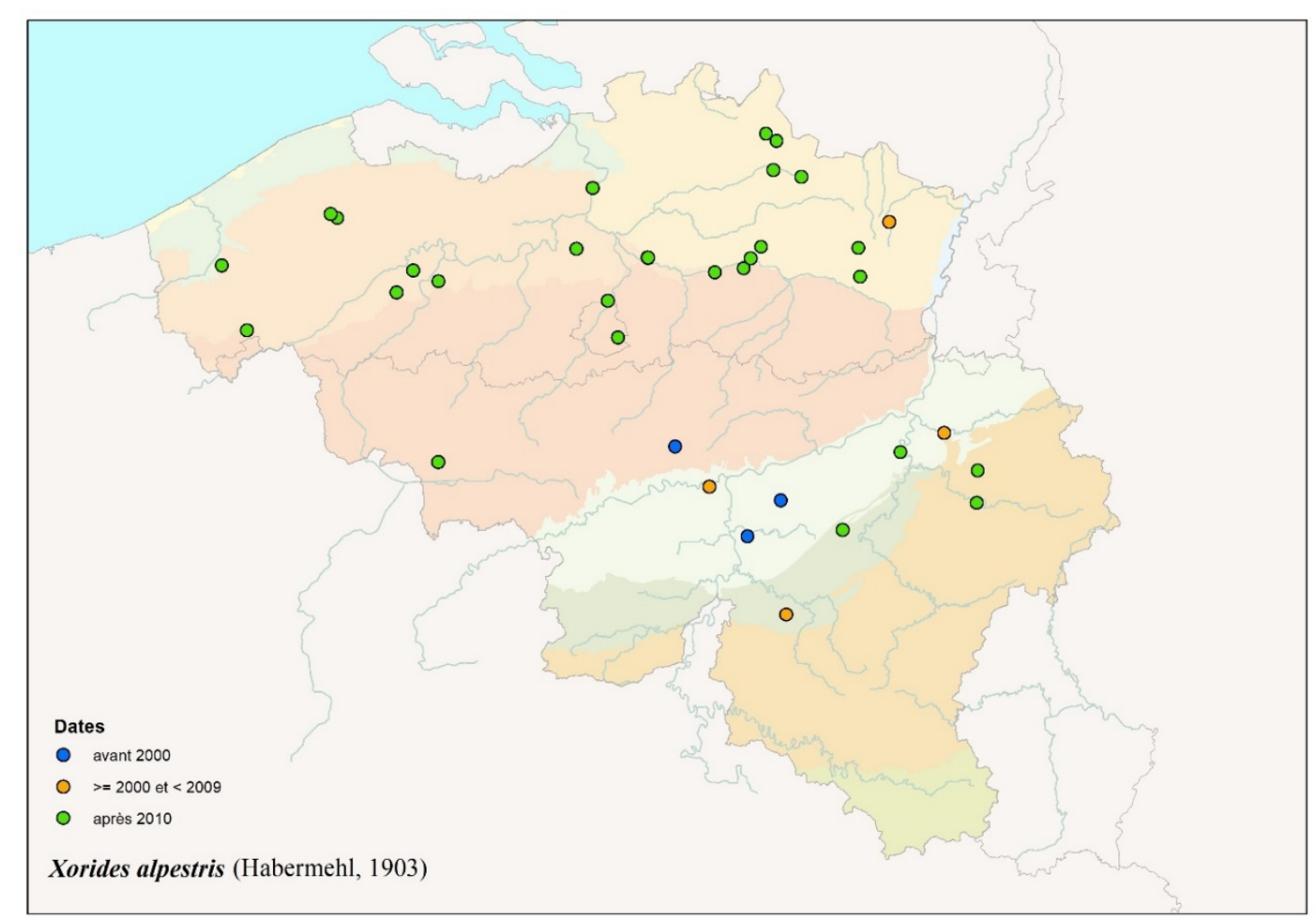

Figure 6 : Répartition de Xorides alpestris (Habermehl, 1903) en Belgique.

11. Xorides brachylabis (Kriechbaumer, 1889) Belg. sp. nov. (Figure 7 et 8)

Matériaux : Esneux, Domaine du Rond-Chêne, piège à vitre au pied de Picea abies, 15-30.iv.2020, 1ठ, 1 , Libert det.

Autres données : Mol, sur Picea abies, 19.vii.2020, 19, Verheyde det. ; Retie, sur Picea abies, 8.v.2020, 1ㅇ, Verheyde det. ; Zillebeke, sur Larix sp., 24.vi.2019, 1 , , Varga \& Verheyde det.

Note: Cette espèce est très récemment apparue chez nous, les premières données remontant à 2019 seulement. Il s'agit d'une espèce globalement rare. Elle est associée au genre Tetropium Kirby (Coleoptera : Cerambycidae) dont elle parasite les larves (Schimitschek, 1929; Chrystal \& Skinner, 1931). Quatre espèces appartenant à ce genre sont présentes en Europe dont trois se retrouvent en Belgique : Tetropium castaneum (Linnaeus, 1758), Tetropium fuscum (Fabricius, 1787) et Tetropium gabrieli Weise, 1905 (Muylaert, 1990). Toutes parasitent les troncs de divers conifères (épicéas, pins, sapins, mélèzes) mais $T$. gabrieli semble inféodé aux mélèzes uniquement (Villiers, 1978). T. castaneum est de loin l'espèce la plus fréquente en Belgique, essentiellement dans le sud du pays, suivie de T. gabrieli de répartition plus éparse sur l'ensemble du territoire (Muylaert, 1990). Seule la troisième espèce, T. fuscum, plus rare, a été trouvée au Rond-Chêne (B. Moucheron, in litt.). 


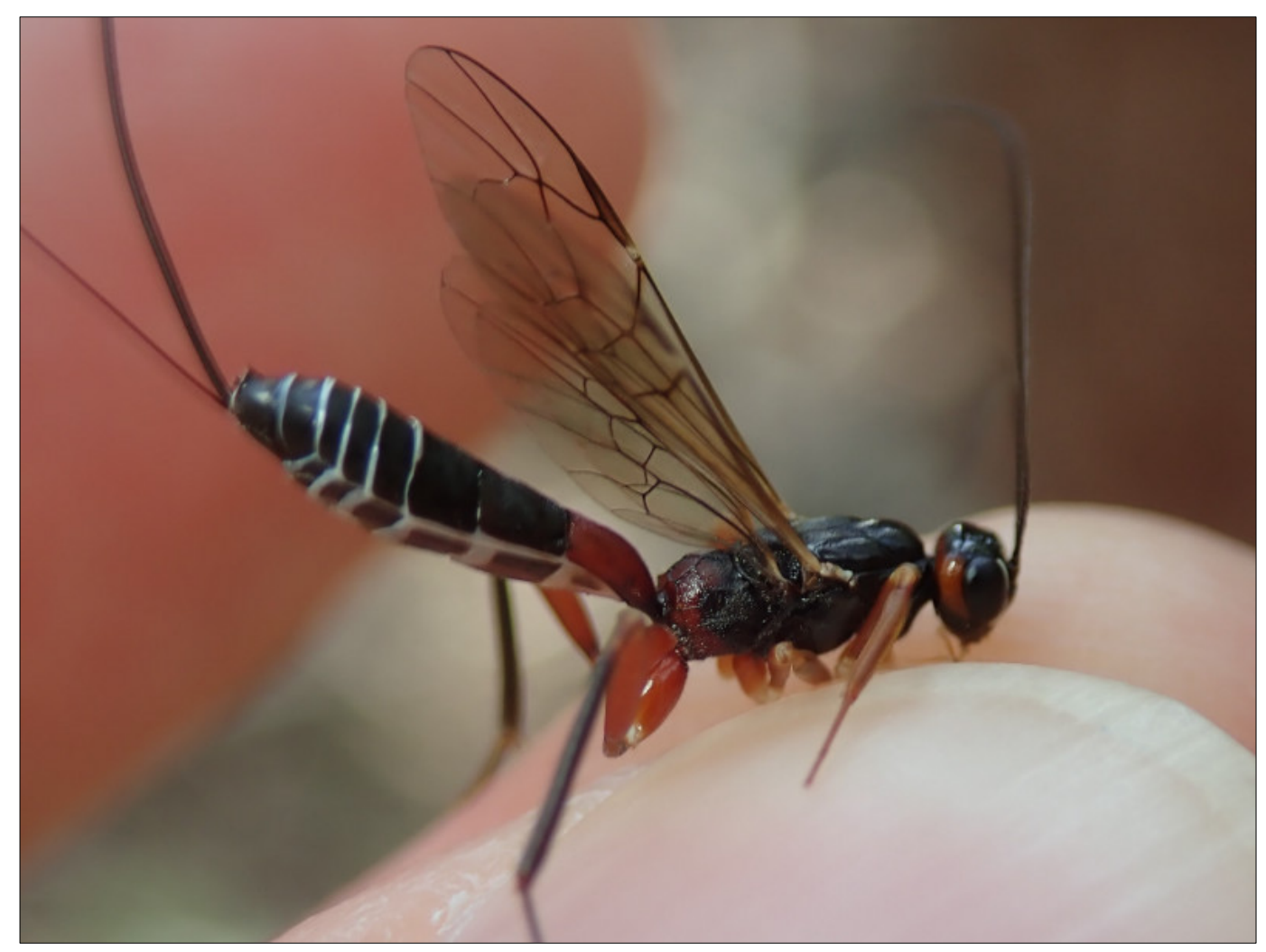

Figure 7 : Xorides brachylabis (Kriechbaumer, 1889), habitus femelle. (Cliché : (C) Juul De Witte, observations.be)

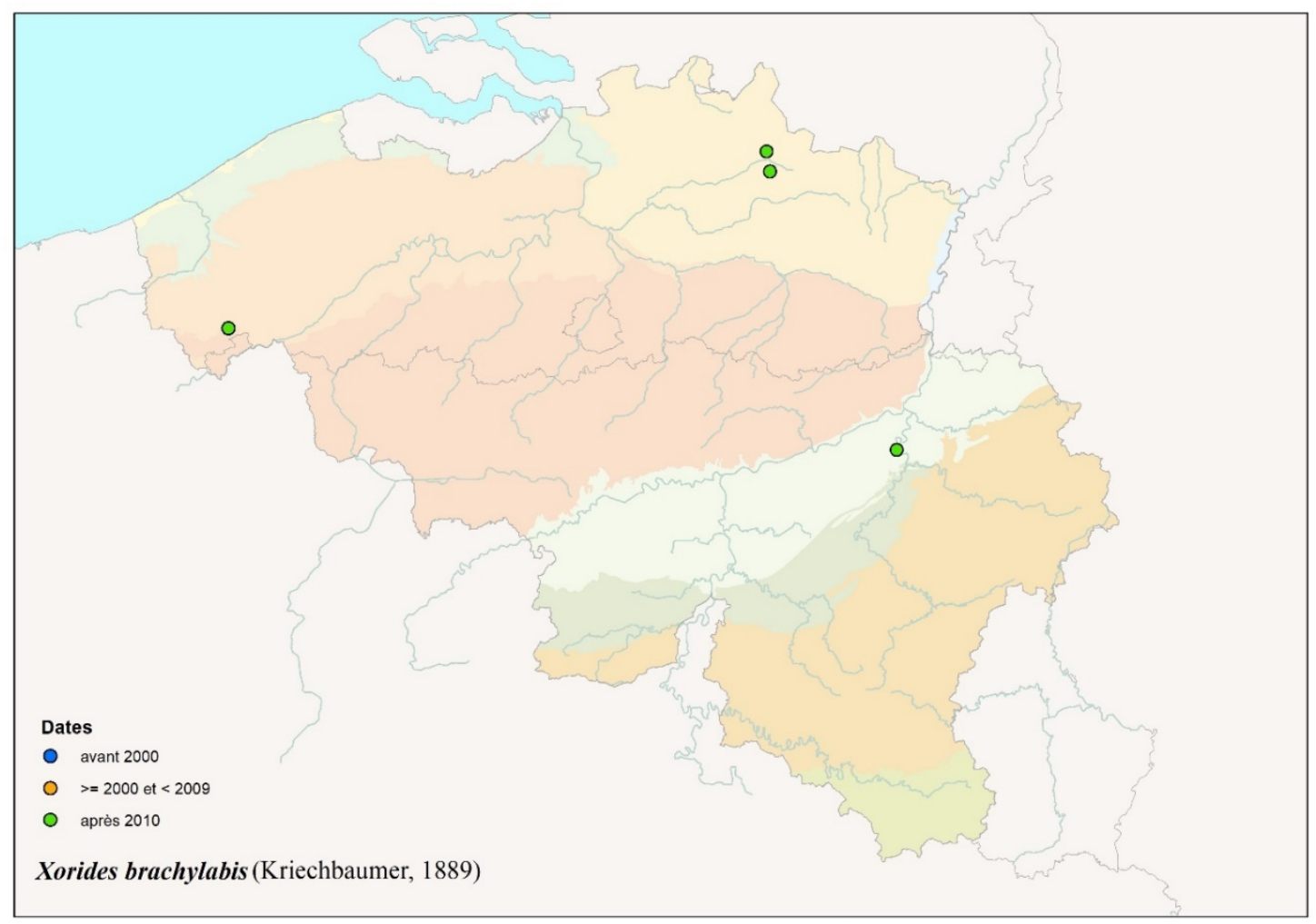

Figure 8 : Répartition de Xorides brachylabis (Kriechbaumer, 1889) en Belgique.

12. Xorides rufipes (Gravenhorst, 1829) Belg. sp. nov. (Figure 9) 
Matériaux : Esneux, Domaine du Rond-Chêne, piège à vitre au pied de Picea abies, 15-31.v.2020, 1 , Libert det.

Autres données : Auderghem, Jardin Massart, piège Malaise, 18-26.vi.2015, 1§, Libert det.; Hanret, 10.viii.2007, 19, Libert det.; Oudenaarde, Bos t'Ename, piège Malaise, 23.viii-9.ix.2015, 19, Libert

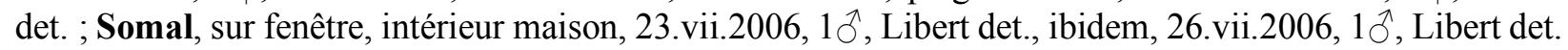
Note: Xorides rufipes (Gravenhorst, 1829) appartient au sous-genre Moerophora Förster facilement reconnaissable à la présence d'une petite dent à l'extrémité des trochantellis antérieurs. Les espèces appartenant à ce sous-genre ont été revues par Jacek Hilszczanski (2000). L'espèce parasite le Coléoptère Cerambycidae Rhagium inquisitor Linnaeus inféodé aux conifères et largement répandu dans le sud-ouest de la Belgique (Muylaert, 1990).

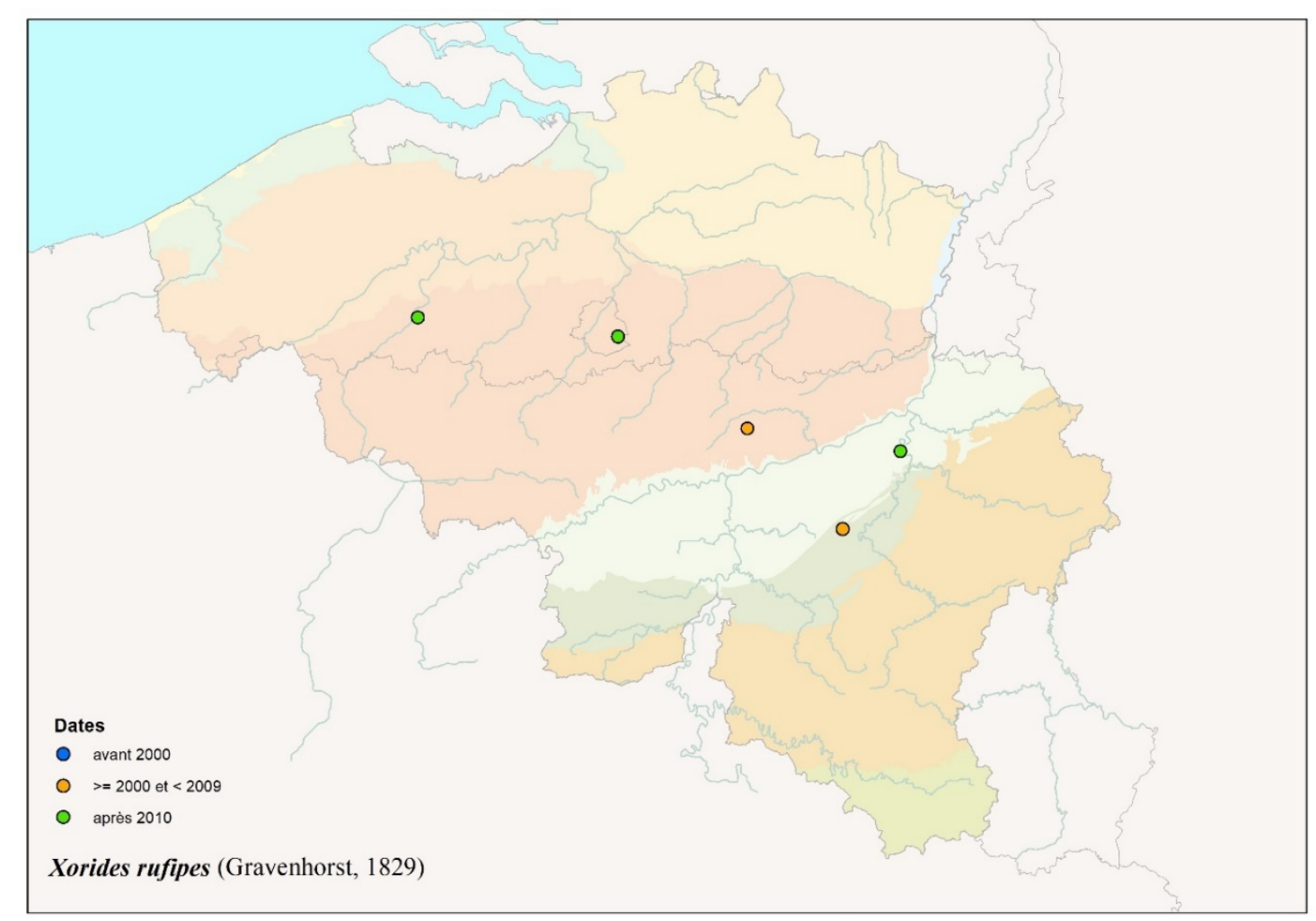

Figure 9 : Répartition de Xorides rufipes (Gravenhorst, 1829) en Belgique. 


\section{REMERCIEMENTS}

Nous remercions Monsieur Bernard Moucheron du Domaine du Rond-Chêne pour avoir eu la gentillesse de nous soumettre les matériaux pour cette étude ainsi que pour nous savoir fourni une photo du piège à vitre en place. Merci à Jean-Luc Vago qui a eu l'immense amabilité de réaliser pour nous plusieurs clichés des insectes capturés. Nous remercions également tous les responsables de collections pour nous avoir accueilli dans leurs institutions respectives. Un merci particulier à Kees van Achterberg pour ses conseils toujours pertinents ! Enfin, merci à Raymond Wahis pour sa relecture attentive d'une version antérieure du manuscrit.

\section{BIBLIOGRAPHIE}

Campadelli G. \& Scaramozzino P. L., 1994. Imenotteri parassitoidi di insetti xilofagi in Romagna. Bollettino dell'Istituto di Entomologia della Universita degli Studi di Bologna, 48, 115-121.

Čapek M., 1958. Revision der europäischen Arten der Gattung Cosmophorus Ratz. (Hym. Braconidae). Acta Entomologica Musei Nationalis Pragae, 32, 151-169.

Chrystal R. N. \& Skinner E. R., 1931. Studies on the biology of Xylonomus brachylabris Kr. and X. irrigator F., parasites of the larch longhorn beetle, Tetropium gabrieli Wiese. Forestry, 5, 21-33.

Hedqvist K. J., 1998. Bark beetle enemies in Sweden II. Braconidae (Hymenoptera). Entomologica scandinavica Supplement, 52, 1-88.

Hilszczanski J., 2000. European species of subgenus Moerophora Foerster of Xorides Latreille (Hymenoptera: Ichneumonidae: Xoridinae), with descriptions of two new species. Insect Systematics \& Evolution, 31, 247-255.

Hilszczanski J., 2002. Polish xoridines and their host associations (Hymenoptera: Ichneumonidae: Xoridinae). In: Melika G. \& Thuroczy C., Editors. Parasitic wasps: evolution, systematics, biodiversity and biological control, Budapest, pp. 294-298.

Horstmann K., 1980. Revision der europäischen Arten der Gattung Rhimphoctona Förster (Hymenoptera, Ichneumonidae). Nachrichtenblatt der Bayerischen Entomologen, 29, 17-24.

Jacobs J. C. \& Tosquinet J., 1897. Catalogue des Ichneumonides de la Belgique appartenant au groupe des Pimplides. Annales de la Société Entomologique de Belgique, 41, 274-328.

Libert P.-N. \& van Achterberg C., 2004. First record of the genus Atanycolus Foerster (Hymenoptera : Braconidae : Braconinae) from the Benelux. Bulletin de la Société royale belge d'Entomologie, 140, 95-96.

Luo Y.-Q. \& Sheng M.-L., 2010. The species of Rhimphoctona (Xylophylax) (Hymenoptera: Ichneumonidae: Campopleginae) parasitizing woodborers in China. Journal of Insect Science, 10(4), 1-9.

Marshall T. A., 1888. Les Braconides. In: André E. (ed.), Species des Hyménoptères d'Europe et d'Algérie. Tome 4. 609 pp.

Muylaert A., 1990. Faune de Belgique : Longicornes (Cerambycidae). Institut royal des Sciences naturelles de Belgique, Bruxelles, 139 pp.

Penigot W. \& Mallet B., 2020. Xorides alpestris (Habermehl, 1903), un nouveau Xorides pour la faune de France (Hymenoptera Ichneumonidae). L'Entomologiste, 76(4), 201-204.

Schimitschek E., 1929. Zur Lebensgechichte und Lebensgemeinschaft von Tetropium gabrieli Weise und Tetropium fuscum F. Zentralblatt für das Gesamte Forstwesen, 55, 369-388.

Šedivý J., 1958. Faunistische und taxonomische Bemerkungen zu den Ichneumoniden der Tschechoslowakei, Pimplinae, I. Acta Faunistica Entomologica Musei Nationalis Pragae, 3, 97-108.

Staverløkk A., Ødegaard F. \& Hansen L.O., 2020. The genus Cosmophorus Ratzeburg, 1848 (Hymenoptera, Braconidae, Euphorinae) in Norway. Norwegian Journal of Entomology, 67, 31-38.

Thirion C., 2005. Liste provisoire des Ichneumonidae de Belgique et du Grand-Duché de Luxembourg (Hymenoptera). Contribution n ${ }^{\circ}$ 1. Notes fauniques de Gembloux, 55, 11-42.

Uhthoff-Kaufmann R. R., 1990a. The occurrence of the subfamily Aseminae (Col.: Cerambycidae) in the British Isles. Entomologist's Record, 102, 55-63. 
Uhthoff-Kaufmann R. R., 1990b. The occurrence of the Callidini tribe (Col.: Cerambycidae) in the British Isles. Entomologist's Record, 102, 161-166.

Uhthoff-Kaufmann R. R., 1991. The genus Pogonocherus Zett. (Col.: Lamiidae) in the British Isles. Entomologist's Record, 103, 243-246.

van Achterberg C., 1987. Revision of the European Helconini (Hymenoptera, Braconidae: Helconinae). Zoologische Mededelingen, 61, 263-285.

van Achterberg C., 2013. Hymenoptera Ichneumonoidea. Fauna Europaea version 2017.06, https://fauna-eu.org (consulté le 5/01/2021).

van Achterberg C. \& Shaw M. R., 2016. Revision of the western Palaearctic species of Aleiodes Wesmael (Hymenoptera, Braconidae, Rogadinae). Part 1: Introduction, key to species groups, outlying distinctive species, and revisionary notes on some further species. ZooKeys, 639, 1-164. Doi: 10.3897/zookeys.639.10893

van Achterberg C., Shaw M. R. \& Quicke D. L. J., 2020. Revision of the western Palaearctic species of Aleiodes Wesmael (Hymenoptera, Braconidae, Rogadinae). Part 2: Revision of the A. apicalis group. ZooKeys, 919, 1-259. Doi: $10.3897 /$ zookeys.919.39642

Varga O., 2014a. Study of Xoridinae (Hymenoptera: Ichneumonidae) in the Ukrainian Carpathians. Genera Odontocolon Cushman and Ischnoceros Gravenhorst. Proceedings of the Russian Entomological Society. St Petersburg, 85(1), 143-150.

Varga O., 2014b. A review of the genus Xorides Latreille, 1809 (Hymenoptera, Ichneumonidae, Xoridinae) in the Ukrainian Carpathians. Journal of Insect Biodiversity, 2(7); 1-9.

Varga O., 2017. A review of the genus Rhimphoctona Förster, 1869 (Hymenoptera: Ichneumonidae: Campopleginae) from the Ukrainian Carpathians, with a key to European species. Zootaxa, 4263(2), 387-394. Doi : $\underline{10.11646 / \text { zootaxa.4263.2.12 }}$

Verheyde F., De Ketelaere A., De Witte J. \& Smets W., 2020. Parasitaire wespen als biodiversiteitsindicatoren (Hymenoptera: Siricidae, Ichneumonidae, Ibaliidae). Natuur.Focus, 19(4), 172-179.

Villiers A., 1978. Faune des Coléoptères de France. 1. Cerambycidae. Encyclopédie entomologique, Lechevalier, Paris, volume 52, $597 \mathrm{pp}$.

von Dalla Torre K. W., 1898. Catalogus Hymenopterorum. Volumen IV. Braconidae. Guilelmi Engelmann. Lipsiae. $323 \mathrm{pp}$.

Yu D. S., van Achterberg C. \& Horstmann K., 2012. World Ichneumonoidea 2011 - Taxonomy, Biology, Morphology and Distribution. DVD/CD. Taxapad. Vancouver, Canada. www.taxapad.com.

\section{(31 Réf)}

